\title{
"Ab Initio" Studies of Hydrogen-Enhanced Oxygen Diffusion in Silicon
}

\author{
R. B. Capaz ${ }^{1}$, L. V. C. Assali ${ }^{2}$, L. C. Kimerling ${ }^{3}$, K. Cho ${ }^{4}$, and J. D. Joannopoulos ${ }^{5}$ \\ ${ }^{1}$ Instituto de Física, Universidade Federal do Rio de Janeiro, \\ Caixa Postal 68528, Rio de Janeiro, RJ 21945-970, Brazil \\ ${ }^{2}$ Instituto de Física, Universidade de São Paulo, \\ Caixa Postal 66318, 05315-970, São Paulo, SP, Brazil \\ ${ }^{3}$ Department of Materials Science and Engineering, \\ Massachusetts Institute of Technology, Cambridge, Massachusetts 02139 \\ ${ }^{4}$ Mechanical Engineering Department, Stanford University, \\ Stanford, California 94305-3030 \\ ${ }^{5}$ Department of Physics, Massachusetts Institute of Technology, \\ Cambridge, Massachusetts 02139
}

Received February 8, 1999

\begin{abstract}
A novel microscopic mechanism for hydrogen-enhanced oxygen diffusion in p-doped silicon is proposed. A path for joint diffusion of $\mathrm{O}$ and $\mathrm{H}$ is obtained from an ab-initio molecular dynamics "kick" simulation. The migration pathway consists of a two-step mechanism, with a maximum energy of $1.46 \mathrm{eV}$. This path represents a $0.54 \mathrm{eV}$ reduction in the static barrier when compared with the diffusion of isolated $\mathrm{O}$ in $\mathrm{Si}$, in excellent agreement with experiments.
\end{abstract}

Being the predominant impurity in Czochralskigrown silicon, oxygen has been extensively studied since the early years of semiconductor research[1]. In particular, the diffusion of oxygen in silicon is extremely important from a technological point-of-view, since it governs both the formation of electric-active oxygen complexes ("thermal donors")[2,3] and the precipitation of $\mathrm{SiO}_{2}$ in silicon [1].

The ground-state configuration of a single oxygen in silicon is a bent bond-center $(B C)$ interstitial $\left(\mathrm{O}_{i}\right)$. Diffusion of $\mathrm{O}_{i}$ in silicon occurs by jumps between neighboring $B C$ sites with a measured migration energy of 2.53-2.56 eV [4]. Theoretical calculations [6, 7] have been performed within the framework of transition state theory (TST) [5], in which the migration energy is expressed as the energy difference between two static configurations: the ground-state and the saddlepoint. For the case of $\mathrm{O}_{i}$, the saddle-point is the wellknown "ylid" configuration, with a three-fold coordinated oxygen. These ab-initio pseudopotential, localdensity-functional, supercell calculations give migration energies in the range of 1.8-2.2 eV. Several mechanisms have been proposed to explain this discrepancy with respect to experimental results $[6,7,8]$.

It has been observed that oxygen diffusion in hydrogenated silicon occurs with a much lower activation energy, 1.6-2.0 eV [9, 10,11]. The microscopic mechanism responsible for this enhancement of oxygen diffusion in the presence of hydrogen is still controversial. There are a few theoretical proposals in the literature. Based on Hartree-Fock cluster calculations, Estreicher [12] has proposed that the diffusion process would initiate with $\mathrm{H}$ in a metastable tetrahedral $(T)$ site which would then assist an oxygen jump to a neighboring bond center with an activation energy of $1.25 \mathrm{eV}$. During the process, the $\mathrm{H}$ atom itself would become trapped in a stable $B C$ configuration. This model assumes that $\mathrm{H}$ diffusion in silicon is an out-of-equilibrium process on which the hydrogen atoms jump between adjacent $T$ sites, without actually visiting the $B C$ ground-state. This view is not supported by either $a b$ initio [13] or tightbinding [?] molecular-dynamics (MD) simulations of hydrogen diffusion. Another proposal is due to Jones et al[15], based on local-density-functional (LDF) cluster calculations. In disagreement with Estreicher, they find the anti-bonding $(A B)$ site opposite to the Si-O- 
Si unit to be the ground-state for $\mathrm{H}$ in the presence of $\mathrm{O}$ (as opposed to the BC site). They propose a mechanism for migration on which the $\mathrm{Si}-\mathrm{H}$ backbond becomes stronger as the $\mathrm{O}$ jumps from one $B C$ position to another, therefore lowering the saddle-point energy to $1.4 \mathrm{eV}$. Recently, Ramamoorthy and Pantelides [16] have investigated the relative stability of several $\mathrm{O}+\mathrm{H}$ configurations in silicon at different charge states. They find the $B C$ configuration to be stable for the positive and neutral charge states, while the $A B$ is stable for the negative configuration. A joint migration pathway is proposed for the neutral $B C$ structure on which the oxygen and hydrogen jump together to adjacent $B C$ configurations, with an activation energy of $1.2 \mathrm{eV}$.

In this work, we perform ab initio total-energy calculations and molecular dynamics (MD) simulations to investigate the mechanism for $\mathrm{H}$-enhanced $\mathrm{O}$ diffusion in Si. Our calculations are based on the pseudopotential, local-density functional and supercell approximations. We use the Rappe et al. optimized pseudopotentials [17], which allow converged calculations for oxygen-containing systems with a plane-wave cutoff of only 40 Ry. A cubic supercell with $64 \mathrm{Si}, 1$ $\mathrm{O}$ and $1 \mathrm{H}$ is adopted, allowing atomic relaxation of up to 3rd-nearest-neighbors of both $\mathrm{O}$ and $\mathrm{H}$. ,-point is used for $\mathrm{k}$-point sampling in most of the calculations. Electronic minimizations are performed using the conjugate-gradients technique [18]. We focus our calculations on the $\mathrm{H}^{+}$charge state, for it is the dominant state for isolated hydrogen in p-doped silicon [19, 20].

Initially we search for the ground state of the $\mathrm{O}+\mathrm{H}$ system in silicon. We find the configuration with both atoms at adjacent $B C$ sites to be lower in energy by 0.22 $\mathrm{eV}$ when compared with the metastable $A B$ configuration. In Fig. 1(a) we show our calculated ground-state geometry and electronic density, and in Table I some calculated bond lengths and bond angles are presented.

We then proceed to investigate the migration pathway. We seek for a pathway which represents a joint diffusion of the $\mathrm{O}+\mathrm{H}$ complex, i.e., on which the final configuration is equivalent to the initial ground-state configuration, except by a translation of both atoms in the jump direction. A rigorous calculation of the saddlepoint for the joint migration is a formidable task, due to the many degrees of freedom involved in the problem.
Therefore, we try a dynamical approach: The $\mathrm{O}$ atom is "kicked" in a direction perpendicular to the $\mathrm{Si}-(\mathrm{O})-\mathrm{Si}$ axis (indicated by the arrow in Fig. 1(a)) with a $2.0 \mathrm{eV}$ kinetic energy and then we let the system evolve in a MD simulation. We define our calculated energy barrier as the energy of a "saddle-point" given by the configuration of maximum potential energy along the trajectory. If our guess to this initial kick is good enough so that it mimics the actual movement of the $\mathrm{O}$ atom just before a migration jump, the other atoms will respond to it in a way to generate a pathway similar to the lowest energy path. It is important to emphasize that the energy barrier calculated by this procedure will be an upper bound to the true minimum barrier for diffusion [21].

The MD simulations are performed using the Verlet algorithm with a $10^{-3} \mathrm{ps}$ time step. As a result of the simulation, we observe that the $\mathrm{O}$ atom jumps over a barrier of $1.46 \mathrm{eV}$. The "saddle-point" configuration, is shown in Fig. 1(b). After the $\mathrm{O}$ atom overcomes the barrier and the potential energy starts to increase again, the MD simulation is stopped and the system is allowed to relaxed to a new minimum-energy configuration. Surprisingly, this configuration is not equivalent to the initial ground-state, but rather a metastable state. This state is shown in Fig. 1(c). Instead of jumping to the next $\mathrm{BC}$ position, the $\mathrm{H}$ atom remains bonded to the central Si atom. Moreover, the Si-Si bond on which the $\mathrm{O}$ was initially centered is not reformed. Therefore the dynamics of the diffusion jump produces a broken-bond defect in Si. This configuration has an energy of only $0.30 \mathrm{eV}$ above the ground state. Interestingly, this type of defect has been proposed to be generated in the diffusi! on jump of a single $\mathrm{O}$ atom in silicon, but in that case it had an energy of $1.2 \mathrm{eV}$ above the respective ground state [6, 22]. Here, the $\mathrm{H}$ atom plays an important role in saturating one of the Si dangling bonds and therefore greatly reduces the energy of this state. The other Si dangling bond remains and it produces a gap state which is empty in the positive charge state. The electronic density associated to this state is shown in Fig. 2. Some geometrical parameters related to this metastable state are also listed in Table I. 

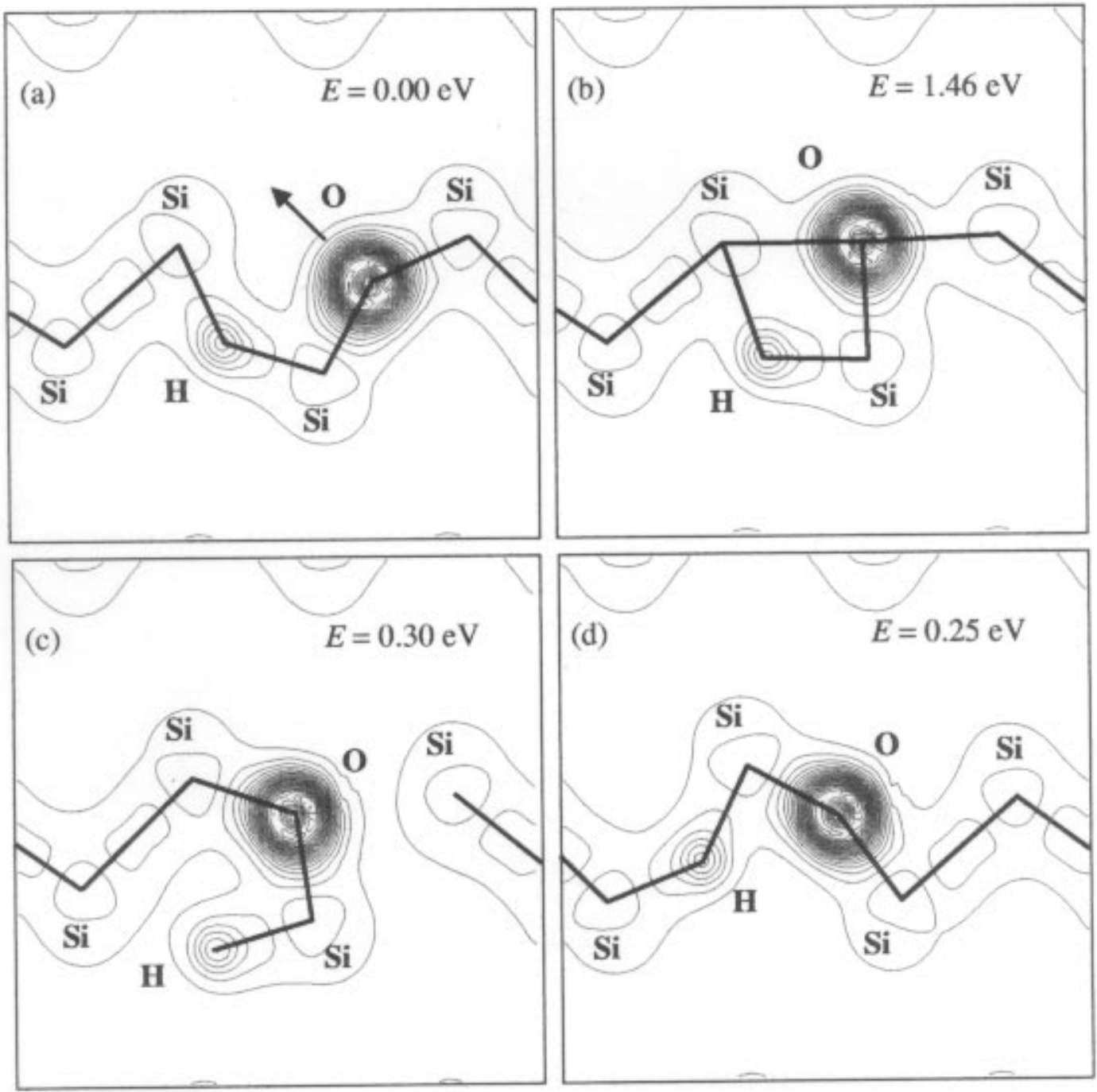

Figure 1. Geometries and electronic densities of four configurations of the $\mathrm{O}+\mathrm{H}$ pair in silicon, on the (110) plane which contains both impurities. Atomic positions are represented by the vertices formed the thick lines. Contours run from 0.25 to 6.25 electrons $/ \AA^{3}$. (a) Initial ground-state configuration, with $B C \mathrm{O}$ and $H$. The arrow indicates the direction of the initial "kick" on the oxygen. (b) "Saddle-point" configuration, defined as the configuration with maximum potential energy (1.46 $\mathrm{eV}$ above the ground state) during the MD simulation. (c) Metastable state with $0.30 \mathrm{eV}$, with H-saturated Si-Si broken bond. (d) Final "ground-state" configuration, with $B C \mathrm{O}$ and H. A true ground-state can be obtained by rotations of $\mathrm{H}$ and $\mathrm{O}$ along the Si-Si axes.

Table I. Some bond lengths and bond angles of the ground- and metastable state configurations of $\mathrm{O}+\mathrm{H}$ in $\mathrm{Si}$, shown in Fig. 1(a) and 1(c), respectively. $\mathrm{Si}_{c}$ denotes the central $\mathrm{Si}$ atom, which is initially bonded to both $\mathrm{O}$ and H.

\begin{tabular}{cc|cc} 
Ground state & & Metastable state & \\
\hline $\mathrm{Si}-\mathrm{O}$ & $1.629 \AA$ & $\mathrm{Si}-\mathrm{O}$ & $1.669 \AA$ \\
$\mathrm{Si}_{c}-\mathrm{O}$ & $1.582 \AA$ & $\mathrm{Si}_{c}-\mathrm{O}$ & $1.630 \AA$ \\
$\mathrm{Si}_{c}-\mathrm{H}$ & $1.586 \AA$ & $\mathrm{Si}_{c}-\mathrm{H}$ & $1.567 \AA$ \\
$\mathrm{Si}-\mathrm{H}$ & $1.645 \AA$ & & \\
$\mathrm{Si}-\mathrm{O}-\mathrm{Si}_{c}$ & $142.7^{\circ}$ & $\mathrm{Si}-\mathrm{O}-\mathrm{Si}_{c}$ & $117.4^{\circ}$ \\
$\mathrm{O}-\mathrm{Si}_{e}-\mathrm{H}$ & $100.6^{\circ}$ & $\mathrm{O}-\mathrm{Si}_{c^{-}} \mathrm{H}$ & $98.0^{\circ}$ \\
$\mathrm{Si}_{c^{-}} \mathrm{H}-\mathrm{Si}$ & $132.2^{\circ}$ & & \\
\hline
\end{tabular}




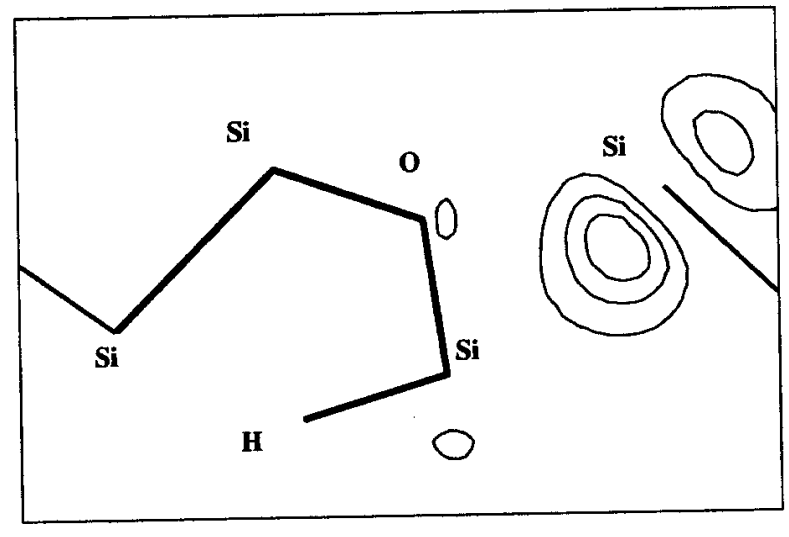

Figure 2. Electronic density for the empty gap state at the metastable configuration. Contours run from 0.02 to 0.10 electrons $/ \AA^{3}$.

In order to complete the joint diffusion jump, the $\mathrm{Si}-\mathrm{H}$ bond has to be broken so that the $\mathrm{H}$ goes to the $\mathrm{BC}$ position and the $\mathrm{Si}-\mathrm{Si}$ bond reforms. We calculate the activation energy for this process by performing constrained total-energy calculations on which the $\mathrm{Si}-\mathrm{H}$ distance is kept fixed at several values between two extreme limits: a initial situation where the $\mathrm{Si}-\mathrm{H}$ distance is small and there is a bond between the two atoms and a final situation where the distance is large and the bond is broken. As a result of these calculations, we find an extra activation energy of just $0.16 \mathrm{eV}$ ( $0.46 \mathrm{eV}$ above the ground state) to bring the system to a final configuration with $\mathrm{H}$ and $\mathrm{O}$ at adjacent $\mathrm{BC}$ sites, shown in Fig. 1(d). This configuration is not exactly equivalent to the ground state (in fact, it has an energy of $0.25 \mathrm{eV}$ above it), but the true ground state can be trivially obtained by rotations of the $\mathrm{O}$ and $\mathrm{H}$ atoms by $180^{\circ}$ around their respective $\mathrm{Si}-\mathrm{Si}$ axes, which are expected to cost very little energy.

Therefore we conclude that $\mathrm{H}$-enhanced $\mathrm{O}$ diffusion in p-doped silicon occurs in a very interesting two-step mechanism. The initial step consists of a jump of the $\mathrm{O}$ atom between two adjacent $\mathrm{BC}$ configurations and the simultaneous formation of an intermediate metastable state corresponding to a H-saturated Si-Si broken-bond defect. In the second step, this defect is annealed and the $\mathrm{H}$ jumps to the stable $\mathrm{BC}$ configuration. It should be interesting to try an experimental detection of this metastable state, but this should be a difficult task due to its small annealing energy barrier. An overall energy barrier of at most $1.46 \mathrm{eV}$ for the combined process is obtained. This is somewhat smaller than the experimental values of 1.6-2.0 eV. This discrepancy is similar to the case of isolated oxygen. Therefore, our barrier should be compared to the total energy of the "ylid" configuration. We calculate this energy to be 2.0 $\mathrm{eV}$ above its respective $B C$ ground state, consistent $\mathrm{w}$ ! ith previous $a b$ initio calculations $[6,7]$. Therefore, the presence of hydrogen is responsible for at least a $0.54 \mathrm{eV}$ reduction in the saddle-point energy, in excellent agreement with experiments. It is now clear the fundamental role played by $\mathrm{H}$ during the diffusion process: it serves both to "open up" a Si-Si bond on which the $\mathrm{O}$ atom will jump into and, more importantly, to decrease the energy of an important intermediate state during the diffusion jump by saturating a Si broken bond. This behavior is quite typical of hydrogen in semiconductors, therefore we believe that hydrogen may behave in similar ways in assisting diffusion jumps of other impurities in semiconductors.

\section{Acknowledgments}

This work is partially supported by Brazilian agencies Conselho Nacional de Desenvolvimento Científico e Tecnológico (CNPq), Programa de Apoio a Núcleos de Excelência (PRONEX-MCT), Fundação de Amparo à Pesquisa do Estado do Rio de Janeiro (FAPERJ) e Fundação de Amparo à Pesquisa do Estado de São Paulo (FAPESP).

\section{References}

[1] Oxygen in Silicon, edited by F. Shimura, Semiconductors and Semimetals Vol. 42 (Academic Press, New York, 1994).

[2] U. Gösele and T. Y. Tan, Appl. Phys. A 28, 79 (1982).

[3] J. W. Corbett, P. Deák, and R. Wu, Mater. Sci. Forum 38-41, 579 (1989).

[4] P. D. Southgate, Proc. Phys. Soc. (London) 36, 385 (1960); C. Haas, J. Phys. Chem. Solids 15, 108 (1960); G. D. Watkins, J. W. Corbett, and R. S. McDonald, J. Appl. Phys. 53, 7097 (1982); S.-T. Lee and D. Nichols, Appl. Phys. Lett. 47, 1001 (1985); J. C. Mikkelsen, Jr., Mat. Res. Soc. Symp. Proc. 59, 19 (1986).

[5] G. H. Vineyard, J. Phys. Chem. Solids 3, 121 (1957).

[6] M. Needels, J. D. Joannopoulos, Y. Bar-Yam, S. T. Pantelides, and R. H. Wolfe, in Defects in Materials, edited by P. D. Bristowe, J. E. Epperson, J. E. Griffith, and Z. Liliental-Weber, MRS Symposia Proceedings 209 (Materials Research Society, Pittsburgh, 1991), p.103.

[7] M. Ramamoorthy and S. T. Pantelides, Phys. Rev. Lett. 76, 267 (1996).

[8] Z. Jiang and R. A. Brown, Phys. Rev. Lett. 74, 2046 (1995).

[9] R. Murray, Physica B 170, 115 (1991). 
[10] S. A. McQuaid, R. C. Newman, J. H. Tucker, E. C. Lightowlers, A. Kubiak, and M. Goulding, Appl. Phys. Lett. 58, 2933 (1991).

[11] H. J. Stein and S. Hahn, J. Appl. Phys. 75, 3477 (1994).

[12] S. K. Estreicher, Phys. Rev. B 41, 9886 (1990).

[13] F. Buda, G. L. Chiarotti, R. Car, and M. Parrinello, Phys. Rev. Lett. 63, 294 (1989).

[14] G. Panzarini and L. Colombo, Phys. Rev. Lett. 73, 1636 (1994).

[15] R. Jones, S. Öberg, and A. Umerski, Materials Science Forum 83-87, 551 (1992).

[16] M. Ramamoorthy and S. T. Pantelides, Solid State Comm. 106, 243 (1998).

[17] A. M. Rappe, K. M. Rabe, E. Kaxiras, and J. D. Joannopoulos, Phys. Rev. B41, 1227 (1990).
[18] M. C. Payne, M. P. Teter, D. C. Allan, T. A. Arias, and J. D. Joannopoulos, Rev. Mod. Phys. 64, 1045 (1992).

[19] C. H. Seager and R. A. Anderson, Appl. Phys. Lett. 53, 1181 (1988).

[20] C. G. Van de Walle, Y. Bar-Yam, and S. T. Pantelides, Phys. Rev. Lett. 60, 2761 (1988).

[21] In fact, a trajectory generated by this molecular dynamics would approach the minimum energy trajectory if the diffusing atom had a mass much greater than the mass of the host atoms, so that the latter would respond almost instantaneously to the movement of the diffuser.

[22] A. Dal Pino, Jr., M. Needels, and J. D. Joannopoulos, Phys. Rev. B 45, 3304 (1992). 